\title{
Reproducibility and Clinical Validation of Automated Habenula Segmentation via deep Learning in Major Depressive Disorder with 7 Tesla MRI
}

\section{Sang-Heon Lim}

Gachon Advanced Institute for Health Sciences and Technology (GAIHST)

Jihyun Yoon

Chungbuk National University Hospital

Young Jae Kim

Gachon University

Chang-Ki Kang

Gachon University

\section{Seo-Eun Cho}

Gachon University Gil Medical Center

\section{Kwang Gi Kim}

Gachon Advanced Institute for Health Sciences and Technology (GAIHST)

Seung-Gul Kang ( $\nabla$ kangsg@gachon.ac.kr)

Gachon University Gil Medical Center

\section{Research Article}

Keywords: habenula , psychiatric diseases, major depressive disorder (MDD)

Posted Date: February 2nd, 2021

DOl: https://doi.org/10.21203/rs.3.rs-170039/v1

License: (9) This work is licensed under a Creative Commons Attribution 4.0 International License.

Read Full License 


\section{Reproducibility and clinical validation of automated}

\section{habenula segmentation via deep learning in major}

\section{depressive disorder with 7 Tesla MRI}

Sang-Heon Lim ${ }^{1,2, \dagger}$, Jihyun Yoon ${ }^{3, \dagger}$, Young Jae Kim², Chang-Ki Kang ${ }^{4}$,

Seo-Eun $\mathrm{Cho}^{5}$, Kwang Gi Kim ${ }^{1,2, *} \&$ Seung-Gul Kang ${ }^{5, *}$

${ }^{1}$ Department of Health Sciences and Technology, Gachon Advanced Institute for Health

Sciences and Technology (GAIHST), Gachon University, Seongnam-si, Republic of Korea;

${ }^{2}$ Department of Biomedical Engineering, Gil Medical Center, College of Medicine, Gachon

University, Republic of Korea;

${ }^{3}$ Department of Family Medicine, Chungbuk National University Hospital, Cheongju,

Republic of Korea;

${ }^{4}$ Department of Radiological Science, College of Health Science, Gachon University,

Incheon, Republic of Korea;

${ }^{5}$ Department of Psychiatry, Gil Medical Center, Gachon University College of Medicine,

Incheon, Republic of Korea;

* These authors contributed equally to this work as corresponding authors: Seung-Gul Kang, kangsg@gachon.ac.kr; Kwang Gi Kim,kimkg@gachon.ac.kr

$\dagger$ These authors contributed equally to this work as first authors. 


\begin{abstract}
The habenula is one of the most important brain regions for investigating the etiology of psychiatric diseases such as major depressive disorder (MDD). However, the habenula is challenging to delineate with the naked human eye in brain imaging due to its low contrast and tiny size, and the manual segmentation results vary greatly depending on the observer. Therefore, there is a great need for automatic quantitative analytic methods of the habenula for psychiatric research purposes. Here we propose a fully-automated segmentation and volume estimation method for the habenula in 7 Tesla magnetic resonance imaging based on a novel fully convolutional network. The proposed method, using the data of 69 participants (33 patients with MDD and 36 normal controls), achieved an average precision, sensitivity, and dice similarity coefficient of $0.869,0.865$, and 0.852 , respectively, in the automated segmentation task. Moreover, the intraclass correlation coefficient reached 0.870 in the volume estimation task. This study demonstrates that this deep learning-based method can provide accurate and quantitative analytic results of the habenula. By providing rapid and quantitative information on the habenula, we expect our proposed method will aid future psychiatric disease studies.
\end{abstract}




\section{Introduction}

The habenula $(\mathrm{Hb})$ is a paired epithalamic structure adjacent to the dorsomedial thalamus and the third ventricle [1] that can be divided into distinct portions via different cellular morphological features. It integrates information received from the cerebral and limbic cortex and provides forebrain control over the activity of ascending monoaminergic projections from the brainstem [2]. Additionally, based on previous studies of $\mathrm{Hb}$ function, the $\mathrm{Hb}$ is involved in the pathogenesis of psychiatric disorders such as major depressive disorder (MDD) [3, 4]. Compared to normal controls (NCs), the $\mathrm{Hb}$ volume of patients with MDD showed atrophy in a post-mortem study [5].

According to previous post-mortem and structural imaging studies, the average volume of the human $\mathrm{Hb}$ is $15-30 \mathrm{~mm}^{3}[5,6]$. Several studies have reported comparing the volume of the $\mathrm{Hb}$ between patients with a psychiatric disorder and NCs: volume comparison among patients with different stages of MDD and NCs [7]; among medicated and unmedicated MDD patients, bipolar disorder patients, and NCs [8]; and among medicated and unmedicated patients with MDD and NCs [9]. The majority of previous human Hb volumetric studies have used manual segmentation to determine $\mathrm{Hb}$ volumes [7-10]. However, these conventional manual-based approaches are time-consuming and laborious, particularly with extensive datasets, and it is challenging to accurately produce the segmented masks due to the anatomical characteristics of the $\mathrm{Hb}$. Thus, manual segmentation results of the $\mathrm{Hb}$ by different observers have large deviations and it is difficult to determine which fit the gold standard. To overcome this problem, two examiners trace the individual region and the reliability of their results are evaluated with an intraclass correlation [11]. Yet, this method is still time-consuming for the tracers. Overall, accurate $\mathrm{Hb}$ segmentation for quantitative analysis is still a challenging task. An accurate and 
quick $\mathrm{Hb}$ segmentation method might be a fundamental step in medical treatment, such as deep brain stimulation and neurosurgery, for targeting $\mathrm{Hb}$ sub-regions related to psychiatric diseases in the future $[12,13]$.

For this reason, a couple of semi- or fully-automatic $\mathrm{Hb}$ segmentation approaches have been reported: 1) reproducibility of a myelin content-based $\mathrm{Hb}$ segmentation from 3T magnetic resonance imaging (MRI) using a semi-automatic myelin contrast-based method [14], and 2) a machine learning technique for fully-automatic $\mathrm{Hb}$ segmentation of 1.5T MRI for $\mathrm{Hb}$ volume comparison of patients with bipolar disorder and schizophrenia with healthy controls [15]. Since those studies performed image processing such as intensity-based threshold and image registration $[14,15]$, there remain limitations in their ability to reliably perform automatic $\mathrm{Hb}$ segmentation in large MRI datasets. Accordingly, the development of accurate methods for a fully-automated $\mathrm{Hb}$ segmentation of 7T MRI in patients with depressive disorder is necessary However, research on automatic analytic methods using a deep learning approach in the depressive disorder research field is currently scarce.

Recently, demonstrated as a powerful tool for semantic segmentation, deep learning methods based on convolutional neural networks can accommodate large annotated datasets and computational resources compared with traditional segmentation techniques [16, 17]. Moreover, various studies have reported regional segmentation of the human brain and their performance using u-net based novel fully convolutional networks (FCNs) [18, 19]. Nevertheless, there are no such reported cases of deep learning approaches for automated $\mathrm{Hb}$ segmentation. Thus, we developed a deep learning-based method for fully-automated $\mathrm{Hb}$ segmentation using high-resolution 7T MRI and assessed the clinical utility of this method using brain images of patients with MDD and NCs for the validation of our deep learning approach. 
Although 7T MRI is an imaging technique suitable for visualizing the $\mathrm{Hb}$, it is still challenging to segment the $\mathrm{Hb}$ accurately using naked eye-based manual segmentation because of its low anatomical contrast and tiny size, resulting in low reliability of segmentation results from different observers. To address this limitation, we designed deep learning networks trained on manual segmentation masks from two different examiners. The final $\mathrm{Hb}$ segmentation results fused the two pre-trained networks' outputs, taking into account both examiners' manually segmented masks.

Additionally, to perform automatic anatomical structure segmentation, it is more efficient to focus on specific areas of the visual scene, picking out only important features of interest, similar to human visual attention, than to examine every part of the brain with the feature aggregation of a deep learning network. The attention u-net was designed for this purpose and has been proposed to simply and accurately segment the pancreas, which occupies a small area in the abdomen $[20,21]$. In this study, therefore, we designed our deep learning networks' architecture based on the attention u-net for robust and accurate $\mathrm{Hb}$ segmentation.

This study aimed to validate the reproducibility of our deep learning-based computer-aided tool via evaluating the automatic $\mathrm{Hb}$ segmentation performance and comparing manual and automated $\mathrm{Hb}$ volume estimation in individuals with MDD and NCs. 


\section{Methods}

Study population. Patients with MDD and NCs ranging from 20 to 65 years of age were recruited from the psychiatric department of Gil Medical Center, Incheon, South Korea. At the screening evaluation, board-certified psychiatrists had structured interviews with the participants to assess their eligibility using the standard diagnostic instrument based on the Diagnostic and Statistical Manual of Mental Disorders, 5th edition (DSM-5) [22]. The severity of psychiatric symptoms of all participants was measured using the Hamilton Depression Rating Scale 17 items (HDRS-17) [23], Beck Depression Inventory (BDI) [24], and Clinical Global Impression Scale (CGI) [24, 25]. This study was conducted in accordance with the Declaration of Helsinki and approved by the Institutional Review Board of the Gil Medical Center (IRB No. GDIRB2018-005), and written informed consent was obtained from all the participants.

The common eligibility criteria for the MDD and NC groups were as follows: (i) no previous abnormal findings on brain imaging; (ii) no intellectual disability, neurocognitive disorders, or history of significant brain injury; (iii) no personality disorder or substance use disorder including alcohol use disorder in the last year; (iv) no major or unstable medical or neurological disorders in the last year; (v) no current serious suicide risk; (vi) right-handedness using the Edinburgh Handedness Test; (vii) not pregnant or lactating; and (viii) no metal material in the body. The NCs were included according to the following additional criteria: (i) no family history of first-degree relatives with a major psychiatric disorder; (ii) no history or symptoms of psychiatric disorders; (iii) no history of taking psychotropics during their lifetime; and (iv) a total score $\leq 6$ on the HDRS-17. The participants who met the DSM-5 diagnostic criteria for MDD [26] were included in the MDD group. The MDD and NC groups were 
matched for age and sex.

Image and label acquisition. Whole-brain sagittal images were acquired using an 8-channel phased-array coil for 7-T MRI (MAGNETOM 7T, Siemens, Erlangen, Germany). To evaluate the possibility of simultaneously recording relaxation times, such as $\mathrm{T} 1$ and $\mathrm{T} 2 *$, the prototype multi-echo magnetization-prepared 2 rapid gradient echoes (MP2RAGE) sequence by Siemens was utilized [27]. The manual segmentation was performed by two well-trained researchers using the T1 map of the participants' 7T MRIs. The researchers manually segmented the target voxels by tracing the $\mathrm{Hb}$, which differed in signal intensity from that of the contiguous brain tissues, using three-dimensional analytic programs (i.e., ImageJ ver. 1.52a). The reliability of the segmentation was inspected using the overlap index ratio (\%) [26].

Experimental overview. Two deep learning networks were trained for automatic $\mathrm{Hb}$ segmentation from the manual segmentation results of two different observers.

Figure 1 shows an overview of the network training and evaluation procedure for the automated $\mathrm{Hb}$ segmentation. $G T_{1}$ and $G T_{2}$ were obtained from two examiners using manual $\mathrm{Hb}$ segmentation. $G T_{1}$ was used to learn as the gold-standard of Network $k_{1}$ and $G T_{2}$ as the goldstandard of Network $k_{2}$. The final automatic segmented region of the $\mathrm{Hb}$ was the intersection of the predicted segmentation results from two different unfamiliar networks in the test dataset. To evaluate the segmentation results, the fusion output label, which was the intersection of the automatic segmentation result, was compared with ground truth (GT), the intersection of $G T_{1}$ and $G T_{2}$.

Preprocessing and experimental setup. We acquired a region of interest mask in the axial plane of the 7T MRI volume (Fig. 2a). The window level and window width were set to clearly observe the Hb on 7T MRI (window level: 1300, window width: 750; converted to an 8-bit 
image) (Fig. 2b). To remove unnecessary brain regions, the images were uniformly cropped to 96 pixels (x-axis) and 128 pixels (y-axis) (Fig. 2c), including the $\mathrm{Hb}$ (Fig. 2d). To train the segmentation network, we divided the total brain MRI data $(n=69)$ by a ratio of $6: 2: 2$ (train:validation:test). A total of 5-folds with the same training, validation, and test set ratio were formed. Accordingly, the performance of the model was evaluated in the whole dataset while shifting the test dataset (Fig. 2e).

Network. The proposed network was designed based on the attention u-net [20,21] which is a modified version of the traditional u-net [29]. The difference between the attention and basic u-net is that the attention u-net includes attention gates (AGs). The AG, located in skipconnection layers of the attention u-net, is a module that helps to optimize the model in segmentation tasks of small and polymorphic regions by using a sigmoid function. The sigmoid function inside the AG has an effect similar to the simultaneous localization and segmentation of the object area via activation considering both the skip-connected layer and the previous layer.

When the 7T MRI was fed into the model (Fig. 3), the significant feature maps were aggregated for $\mathrm{Hb}$ segmentation by the convolution operation. In the feature aggregation process, the feature map, which was reduced in resolution by a repeated pooling operation, was restored to the input image resolution by the up-sampling operation. Up-sampling was performed after the AG operation. The feed-forward procedure of the AG-based up-sampling was as follows:

$$
\begin{gathered}
A G_{\text {in }}=\sigma_{1}\left(W_{\alpha}(x)+W_{\alpha}(g)\right) \\
A G_{\text {out }}=\sigma_{2}\left(W_{s}\left(A G_{\text {in }}\right)\right) \times W_{\alpha}(g)
\end{gathered}
$$




$$
\text { Concatenate }=\operatorname{merge}\left(A G_{\text {out }}, x\right)
$$

Where $\sigma_{1}$ is the rectified linear unit (ReLU) activation function, $\sigma_{2}$ is the sigmoid activation function, $W_{\alpha}(f)$ is a convolutional operation that maintains the number of feature map channels of $f$, and $W_{s}(f)$ is a convolutional operation that aggregates a single-channel from the $f$. The two input features maps, up-sampled feature map $x$ and skip-connected feature map $(g)$, are added, after a convolution operation $W_{\alpha}$. After summation, the added feature map $W_{\alpha}(x)+W_{\alpha}(g)$ is activated by ReLU. The single feature map aggregated by $W_{s}$ is output as an activation map via sigmoid activation. The output of AG is a self-weighted feature map created by multiplying the activation map $\sigma_{2}\left(W_{s}\left(A G_{i n}\right)\right)$ and the skip-connected feature map $W_{\alpha}(g)$. Finally, the concatenate layer is a self-weighted feature map that focuses on the important features for segmentation of the $\mathrm{Hb}$. The concatenate layer, including AG, affects the final network's output by continuing to participate in the subsequent up-sampling operation.

Implementation details. We trained our networks on a single Tesla V100 (32GB) GPU (graphics processing unit). Each network consisted of 1,984,565 parameters. Our networks were trained using the Adam optimizer [30] to jointly minimize the generalized dice loss [31]. We conducted early termination of the training procedure when loss did not improve during 50 epochs. The initial learning rate (LR) was 0.001 , and when the loss did not minimize for 10 epochs, the LR was reduced by a factor of 0.2 . The networks early terminated the training procedure in 100-300 epochs.

Statistics. Demographic data and clinical characteristics were calculated and compared using two-tailed independent t-tests and chi-square tests. The software IBM SPSS Statistics (ver. 21.0) was used and $\mathrm{P}<0.05$ was set as the limit for statistical significance for these analyses.

We obtained the precision, sensitivity, and dice similarity coefficient (DSC) by comparing 
the GT and automatic segmentation result of networks for evaluation in the test set.

To evaluate our network, we calculated the coincidence-rate of the GTs and autosegmentation results. The evaluation was conducted slice-by-slice with binary $2 \mathrm{D}$ images using the following equations:

$$
\begin{gathered}
\text { Precision }=\frac{T P}{T P+F P} \\
\text { Recall }=\frac{T P}{T P+F N} \\
D S C=\frac{2 \times \text { Precision } \times \text { Recall }}{\text { Precision }+ \text { Recall }}
\end{gathered}
$$

The True Positive (TP), False Positive (FP), True Negative (TN), and False Negative (FN) were obtained by comparing the voxels of a GT and fusion segmentation result. Since the whole dataset was divided into 5-fold as a test dataset, we were able to evaluate every slice in our whole dataset (69 participants). Since deep neural networks (DNNs) are dependent on the training and validation set, we designed the training, validation, and test dataset to 5-fold for every test dataset in each fold.

For the validation of clinical applications such as volume analysis, we compared manual and automated $\mathrm{Hb}$ segmentation in participants with MDD and NCs. Therefore, it was necessary to estimate the size of the $\mathrm{Hb}$ volume via 3D volume reconstruction for each participant (see Supplementary Fig. S1). In addition, we divided the total volume of the $\mathrm{Hb}$ into the left and right hemispheres to analyze automatic segmentation performance on each side.

After $3 \mathrm{D}$ reconstruction of the $\mathrm{Hb}$, the intraclass correlation coefficients (ICCs) were calculated from each pair of brain volumes using the automatic and manual segmentation 
methods. Before this analysis, the normalization of the $\mathrm{Hb}$ volumes was performed using total intracranial volume (ICV). The Hb volumes were divided by the ICV for each participant $\left(\frac{H b \text { Volume }}{I C V} \times 100\right)$ to adjust for individual differences in brain size. To assess the inter-rater reliability (i.e., the degree of agreement between the $\mathrm{Hb}$ volumes by automatic and manual segmentation), the ICC method involving the absolute agreement mode, which is sensitive to the differences in the mean values of observations, was used $[32,33]$. The reliability ICC $\left(r_{I C C}\right)$ values were interpreted according to Cicchetti's guidelines [34] as follows: $<0.40$, poor; 0.40 0.59, fair; 0.60-0.74, good; and 0.75-1.00, excellent. SPSS was used for the ICC analysis. 


\section{Results}

Demographics. Supplementary Table S1 shows the demographics of the participants in this study. The age and sex ratio did not significantly differ between the two groups. The years of education and depressive symptom severity measured using the HDRS-17, BDI, and CGI differed significantly between the two groups.

Evaluation of habenula segmentation. The average total number of voxels with automated segmentation for the $\mathrm{Hb}$ out of all voxels $(256 \times 256 \times 208)$ was $24.01 \pm 6.42 \mathrm{~mm}^{3}$ (mean \pm standard deviation), and in the case of manual segmentation it was $24.19 \pm 6.10 \mathrm{~mm}^{3}$. Table 1 shows the performance evaluation of the automated $\mathrm{Hb}$ segmentation. The performance of our network reached a mean precision, sensitivity, and DSC of $0.869,0.865$, and 0.852 , respectively, using 5-fold cross-validation. We also trained a single attention u-net from the intersected GT of the two raters for an ablation study of our proposed network. It achieved a mean precision, sensitivity, and DSC of $0.847,0.789$, and 0.790 , respectively, in 5-fold. In the ablation study, the proposed network achieved a higher sensitivity than did the network that did not consider the two raters' manual segmentation results (see Supplementary Table S2 \& Fig. S2).

Figure 4 shows a cross-sectional view of the segmentation results of several cases. The segmentation performance of the automated segmentation model was highly consistent with GT. The cross-validation results showed that the segmentation performance was similar for all folds, and there was a low standard deviation in each fold.

Intraclass correlation analysis. Table 2 shows the results of intraclass correlation analyses between automated and manual segmentation of the $\mathrm{Hb}$ volume. The mean of the normalized total $\mathrm{Hb}$ volume with automatic segmentation was $16.66 \times 10^{-4} \pm 4.39 \times 10^{-4}$, and the mean of the 
normalized total $\mathrm{Hb}$ volume with manual segmentation was $16.77 \times 10^{-4} \pm 4.05 \times 10^{-4}$. The ICCs calculating the agreement between the $\mathrm{Hb}$ volumes by automatic and manual segmentation were excellent for the MDD group $\left(r_{I C C}=0.818, p<0.001, \mathrm{n}=33\right)$, NC group $\left(r_{I C C}=0.897\right.$, $p<0.001, \mathrm{n}=36)$, and participants overall $\left(r_{I C C}=0.870, p<0.001, \mathrm{n}=69\right)$.

We divided the $\mathrm{Hb}$ volume into the left and right hemispheres to investigate differences of estimated $\mathrm{Hb}$ volumes between automated and manual segmentation. Significant ICCs were obtained for both the left and right $\mathrm{Hb}$. In the left $\mathrm{Hb}$, excellent ICCs were observed in the MDD group $\left(r_{I C C}=0.920, p<0.001\right)$, NC group $\left(r_{I C C}=0.903, p<0.001\right)$, and participants overall $\left(r_{I C C}=0.908, p<0.001\right)$. In the right $\mathrm{Hb}$, we obtained excellent ICC values in the NC group $\left(r_{I C C}=0.819, p<0.001\right)$ and participants overall $\left(r_{I C C}=0.750, p<0.001\right)$, and a good ICC value for the MDD group $\left(r_{I C C}=0.658, p=0.001\right)$. Boxplots illustrating the distribution of the estimated $\mathrm{Hb}$ volumes for automatic and manual segmentation in the MDD and NC groups are presented in Figure 5. 


\section{Discussion}

In this study, we proposed a deep attention u-net-based intersection network for accurate $\mathrm{Hb}$ segmentation and quantitative $\mathrm{Hb}$ analysis. As a result of experiments, the mean precision, mean sensitivity, and mean DSC in the automatic segmentation using the intersection of attention u-net was good in the total participants. Additionally, the ICCs between automatic and manual segmentation of the total $\mathrm{Hb}$ were excellent in all participants, participants with MDD, and NCs. Therefore, we suggest that the proposed approach is suitable for the segmentation of the $\mathrm{Hb}$, which is a brain region tiny in size with low contrast in brain MRI. To the best of our knowledge, this is the first study which presented a fully-automatic $\mathrm{Hb}$ segmentation based on volume estimation method in participants with MDD and NCs using 7T MRI.

In the automatic segmentation procedure, we obtained a mean precision, sensitivity, and DSC of $0.869,0.865$, and 0.852 , respectively, in the whole dataset. In recent years, a couple of studies on automated segmentation of the $\mathrm{Hb}$ have been reported. The first study performed semi- and fully-automated segmentation in 3T MRI of healthy young adults [14], and the second study performed fully-automated segmentation in children, adolescents, and adults with bipolar disorder and schizophrenia [15]. In the first study, the DSC for binary segmentation reached 0.71 for semi-automated segmentation and 0.69 for fully-automated segmentation, and the DSC of the probability map reached 0.74 for both semi- and fully-automated segmentation [14]. In a more recent study that segmented the $\mathrm{Hb}$ with a fully-automated framework, the DSC of the inter-rater reliability tests between manual and automatic segmentation ranged between 0.758 and 0.828 [15]. Although the participants in the previous studies had different clinical characteristics from those in our study, our automatic $\mathrm{Hb}$ segmentation approach seems to be 
more accurate (mean DSC > 0.85) than that of the other studies.

In this study, the $r_{I C C}$ of the total $\mathrm{Hb}$ ranged between 0.818 and 0.897 , depending on the group. In a previous study conducted on healthy young adults, the $r_{I C C}$ for the Hb was 0.62 for semi-automated segmentation and 0.47 for fully-automated segmentation [14], which shows the superiority of our approach. However, the $r_{I C C}$ of the $\mathrm{Hb}$ was different between the groups and hemispheres in our study. Specifically, the $r_{I C C}$ of the left Hb was excellent (0.903-0.920), while the $r_{I C C}$ of the right $\mathrm{Hb}$ was from 0.658 (NC) to 0.819 (MDD). It is difficult to accurately explain why the $r_{I C C}$ for the left and right $\mathrm{Hb}$ were different; however, the asymmetry of the left and right $\mathrm{Hb}$ might be one reason [35].

Another attribute of our approach is that the results of two networks, each trained on GT generated by two different observers, were intersected to output fusion segmentation results. When trained with the intersected GT, the network reached a low mean sensitivity $(0.789)$ compared to that of the single attention-network (0.865). We assume that the single attentionnetwork was not able to fully capture the anatomical context of the $\mathrm{Hb}$ without considering the different viewpoints of two examiners.

Our approach is different from the previous studies for following reasons: first, this is the first fully-automated segmentation study performed in participants with MDD and NCs using high-resolution 7T MRI that can ideally visualize the Hb. Second, a DNNs approach for automatic $\mathrm{Hb}$ segmentation and volume estimation was conducted. We designed a deep learning network based on the attention u-net that was optimized for segmenting small objects (i.e., the $\mathrm{Hb}$ ) of various shapes. Third, since the segmentation was performed by the fusion of the two pre-trained attention u-net using two different GTs, it is believed that a more reliable segmentation was achieved.

The high DSC and reproducibility of the automated segmentation of this study demonstrate 
that the applicability of the DNNs' approach for $\mathrm{Hb}$ volume estimation in 7T MRI is promising. Although the $\mathrm{Hb}$ is considered to be an important brain region in the etiology of major psychiatric disorders, its small size has made it difficult to investigate via neuroimaging. The $\mathrm{Hb}$ is involved in emotional and cognitive processes, having connections to many other areas of the brain (e.g., thalamus, prefrontal cortex, basal ganglia, and brainstem monoaminergic neurotransmitter systems) [36, 37]. Recently, there are many studies focused on the connectivity between the $\mathrm{Hb}$ and other brain regions of interest such as monoamine centers and the thalamus in depression $[38,39]$. However, manual segmentation is time-consuming, highly variable, and the rater must acquire a high level of technical ability and anatomical knowledge for accurate segmentation, which has become a significant barrier to entry into this field of research [15].

Considering that the data acquired through neuroimaging research is gradually increasing and that machine learning techniques are becoming more popular, the automatic segmentation approach in our study is expected to be a useful tool for many future studies. 


\section{Conclusion}

This study presented an intersection network based on attention u-net for a fully-automated segmentation of the $\mathrm{Hb}$ using 7T MRI that performed automatic segmentation and estimated the $\mathrm{Hb}$ volume with high accuracy and reproducibility (i.e., high DSC and correlation coefficients). Although the sample size was not large (69 participants), cross-validation confirmed that reliable $\mathrm{Hb}$ segmentation results can be obtained using our network.

Furthermore, it is expected that the proposed automatic $\mathrm{Hb}$ segmentation method will be useful for future psychiatric neuroimaging studies to facilitate automatic segmentation and volume estimation of the $\mathrm{Hb}$ and other important small brain regions in 7T MRI.

\section{Data availability}

The datasets generated during or analyzed during the current study are available from the corresponding author on reasonable request. 


\section{References}

1. Hikosaka, O., Sesack, S.R., Lecourtire, L. \& Shepard, P.D. Habenula: crossroad between the basal ganglia and the limbic system. Journal of Neuroscience. 28, 11825-11829 (2008).

2. Poller, W.C., et al. Lateral habenular neurons projecting to reward-processing monoaminergic nuclei express hyperpolarization-activated cyclic nucleotid-gated cation channels. Neuroscience. 193, 205-216 (2011).

3. Batalla, A., et al. The role of the habenula in the transition from reward to misery in substance use and mood disorders. Neurosci Biobehav Rev. 80, 276-285 (2017).

4. Lecca, S., Meye, F.J. \& Mameli, M. The lateral habenula in addiction and depression: an anatomical, synaptic and behavioral overview. Eur J Neurosci. 39, 1170-1178 (2014).

5. Ranft, K., et al. Evidence for structural abnormalities of the human habenular complex in affective disorders but not in schizophrenia. Psychol Med. 40, 557-567 (2009).

6. Savitz, J.B., Rauch, S.L. \& Drevets, W.C. Reproduced from Habenula volume in bipolar disorder and major depressive disorder: a high-resolution magnetic resonance imaging study. Mol Psychiatry. 18, $523(2013)$.

7. Carceller-Sindreu, M., et al. Volumetric MRI study of the habenula in first episode, recurrent and chronic major depression. Eur Neuropsychopharmacol. 25, 2015-2021 (2015).

8. Savitz, J.B., et al., Habenula volume in bipolar disorder and major depressive disorder: a highresolution magnetic resonance imaging study. Biol Psychiatry. 69, 336-343 (2011).

9. Schmidt, F.M., et al. Habenula volume increases with disease severity in unmedicated major depressive disorder as revealed by 7T MRI. Eur Arch Psychiatry Clin Neurosci. 267, 107-115 (2017).

10. Lawson, R.P., Drevets, W.C. \& Roiser, J.P. Defining the habenula in human neuroimaging studies. Neuroimage. 64, 722-727 (2013).

11. Pantel, J., et al. A new method for the in vivo volumetric measurement of the human hippocampus 
with high neuroanatomical accuracy. Hippocampus. 10, 752-758 (2000).

12. Akram, H., et al. Subthalamic deep brain stimulation sweet spots and hyperdirect cortical connectivity in Parkinson's disease. Neuroimage. 158, 332-345 (2017).

13. Morris, L.S., et al. Ultra-high field MRI reveals mood-related circuit disturbances in depression: a comparison between 3-Tesla and 7-Tesla. Translational psychiatry. 9, 1-11 (2019).

14. Kim, J.W., et al. Reproducibility of myelin content-based human habenula segmentation at 3 Tesla. Hum Brain Mapp. 39, 3058-3071 (2018).

15. Germann, J., et al. Fully automated habenula segmentation provides robust and reliable volume estimation across large MRI datasets suggesting intriguing developmental trajectories in psychiatric disease. Biol Psychiatry Cogn Neurosci Neuroimaging. 5, 923-929 (2020).

16. Shelhamer, E., Long J. \& Darrell T. Fully convolutional networks for semantic segmentation. IEEE Trans Pattern Anal Mach Intell. 39, 640-651 (2017).

17. LeCun, Y. \& Bengio, Y. Convolutional networks for images, speech, and time series.(ed. Arbib, M.A.). 255-258 (MIT press, 1995).

18. Nguyen, D.M.H., Vu, H.T., Ung, H.Q. \& Nguyen, G.T. 3D-brain segmentation using deep neural network and gaussian mixture model. in proceedings of the IEEE Winter Conference on Applications of Computer Vision. 815-824 (IEEE, 2017)

19. Ding, Z., Han, X. \& Niethammer, M. Votenet +: An Improved Deep Learning Label Fusion Method for Multi-Atlas Segmentation. In proceedings of 2020 IEEE International Symposium on Biomedical Imaging. 363-367 (IEEE, 2020)

20. Oktay, O., et al. Attention u-net: learning where to look for the pancreas. Preprint at, https://arxiv.org/abs/1804.03999 (2018).

21. Anderson, P., et al. Bottom-up and top-down attention for image captioning and visual question answering. In Proceedings of the IEEE conference on computer vision and pattern recognition. 6077-6086 (IEEE, 2018). 
22. First, M., et al. Structured Clinical Interview for DSM-5-Research Version (SCID-5 for DSM-5, Research Version; SCID-5-RV) 1-94 (American Psychiatric Association , 2015).

23. Yi, J.S., et al. Validity and reliability of the Korean version of the Hamilton Depression Rating Scale (K-HDRS). Journal of Korean Neuropsychiatric Association, 44, 456-465 (2005).

24. Han, H.M., et al. Korean standardization study of Beck Depression Inventory in Korea. Journal of The Korean Neuropsychiatric Association. 25, 487-502 (1986).

25. Busner, J. \& Targum, S.D. The clinical global impressions scale: applying a research tool in clinical practice. Psychiatry (Edgmont). 4, 28 (2007).

26. American Psychiatric Association. Diagnostic and statistical manual of mental disorders (DSM5®) (American Psychiatric Association, 2013)

27. Metere, R., Kover, T., Möller, H.E. \& Schäfer, A. Simultaneous quantitative MRI mapping of T1, T2* and magnetic susceptibility with multi-echo MP2RAGE. PloS one. 12, e0169265; 10.1371/journal.pone.0169265 (2017).

28. Haro, J.M., et al., The Clinical Global Impression-Schizophrenia scale: a simple instrument to measure the diversity of symptoms present in schizophrenia. Acta Psychiatr Scand Suppl. 107, 16$23(2003)$.

29. Ronneberger, O., Fischer, P. \& Brox, T. U-net: convolutional networks for biomedical image segmentation. Preprint at, https://arxiv.org/abs/1505.04597 (2015).

30. Kingma, D.P. \& Ba, J. Adam: a method for stochastic optimization. Preprint at, https://arxiv.org/abs/1412.6980v9 (2014).

31. Sudre, C.H., Li, W., Vercauteren, T., Ourselin, S. \& Cardoso, M. Generalised dice overlap as a deep learning loss function for highly unbalanced segmentation. in proceeding of the International Workshop on Deep Learning in Medical Image Analysis. 10553, 240-248 (Springer, 2017).

32. Fisher, R.A. Statistical Methods, Experimental Design, and Scientific Inference (ed. Bennett J.H.) (Oxford University Press , 1990).

33. Fisher, R.A. Statistical Methods for Research Workers. 356 (Oliver and Boyd, 1954). 
34. Cicchetti, D.V. Guidelines, criteria, and rules of thumb for evaluating normed and standardized assessment instruments in psychology. Psychological Assessment. 6, 284-290 (1994).

35. Ahumada-Galleguillos, P., et al. Directional asymmetry in the volume of the human habenula. Brain Struct Funct. 222, 1087-1092 (2017).

36. Benarroch, E.E., Habenula: Recently recognized functions and potential clinical relevance. Neurology. 85, 992-1000 (2015).

37. Boulos, L.J., Darcq, E. \& Kieffer, B.L. Translating the Habenula-From Rodents to Humans. Biol Psychiatry. 81, 296-305 (2017).

38. Ely, B.A., et al. Resting-state functional connectivity of the human habenula in healthy individuals: Associations with subclinical depression. Hum Brain Mapp. 37, 2369-2384 (2016).

39. Luan, S.X., Zhang, L., Wang, R., Zhao, H. \& Liu, C. A resting-state study of volumetric and functional connectivity of the habenular nucleus in treatment-resistant depression patients. Brain Behav. 9, e01229; 10.1002/brb3.1229 (2019). 


\section{Acknowledgements}

This study was supported by a grant from the Korea Health Technology R\&D Project through the Korea Health Industry Development Institute funded by the Ministry of Health and Welfare, Republic of Korea (grant number: HI17C2665). This work was also supported by a National Research Foundation of Korea (NRF) grant funded by the Korean government (MSIT) (grant number: NRF-2020R1A2C1007527) and by the Gyeonggi-do Regional Research Center (GRRC) program of Gyeonggi province (GRRC-Gachon2020(B01), AI-based Medical Image Analysis).

\section{Author contributions}

S.H.L. conducted the deep learning analysis, drafted the manuscript, and generated the figures. J.H.Y. drafted and revised the manuscript. S.G.K. designed the study, recruited the participants, performed statistical analyses, and revised the manuscript. Y.J.K. and C.K.K. advised the study design and automatic and manual segmentation. S.E.C. recruited the participants and performed statistical analyses. K.G.K. designed the study, supervised the deep learning analysis, and revised the manuscript. All authors reviewed the manuscript.

\section{Competing interests}

The authors declare no competing interests. 


\section{Figure legends}

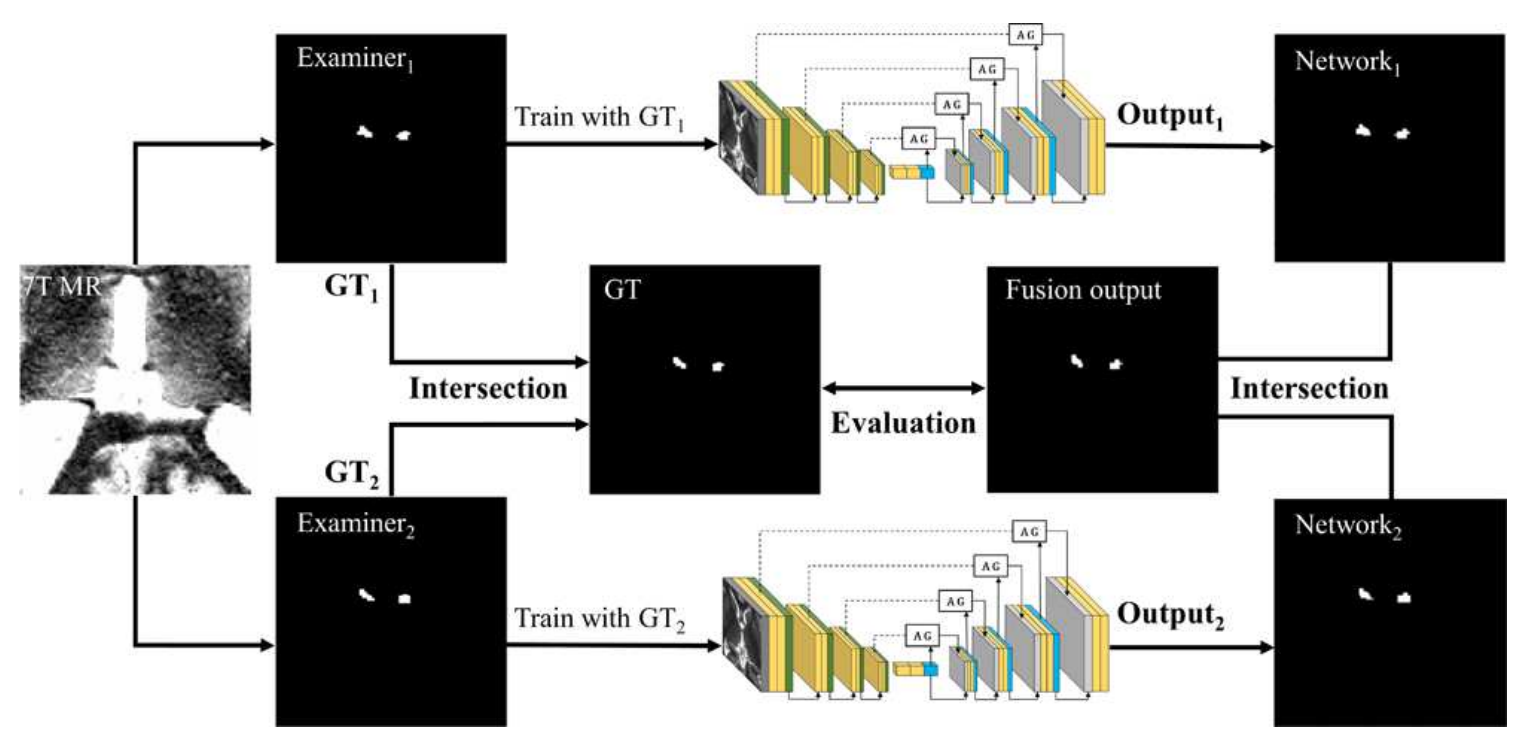

Figure 1. An illustrated overview of the automatic habenula segmentation.

In the network training procedure, two manual segmentation masks were used for the training of two networks, and two segmentation results were obtained. The network evaluation was performed by comparing the intersected GT and fusion output.

Abbreviations: GT, ground truth; MR, magnetic resonance; 7T, 7 Tesla; AG, attention gate. 

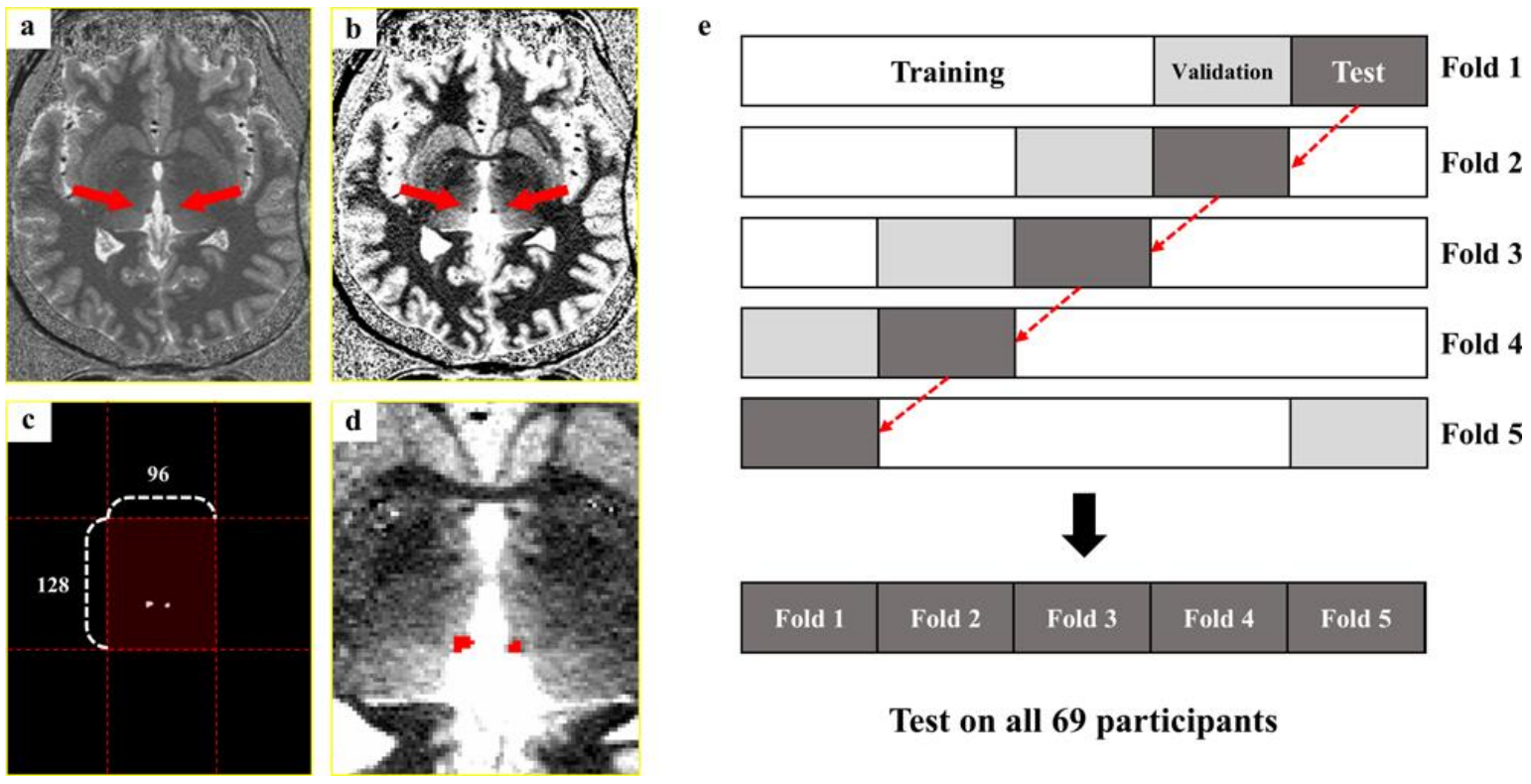

Test on all 69 participants

Figure 2. Preprocessing procedure of 7T MR images (a-d), and experimental setup (e).

(a) Original axial view 7T MRI. (b) Window level (WL) and window width (WW) were set (WL/WW: 1300/750) to observe the Hb. (c) Region of the habenula. (d) Cropped to the specific size. (e) The dataset was divided into 5 folds for testing the segmentation performance on all participants.

Abbreviations: MR, magnetic resonance; 7T, 7 Tesla 

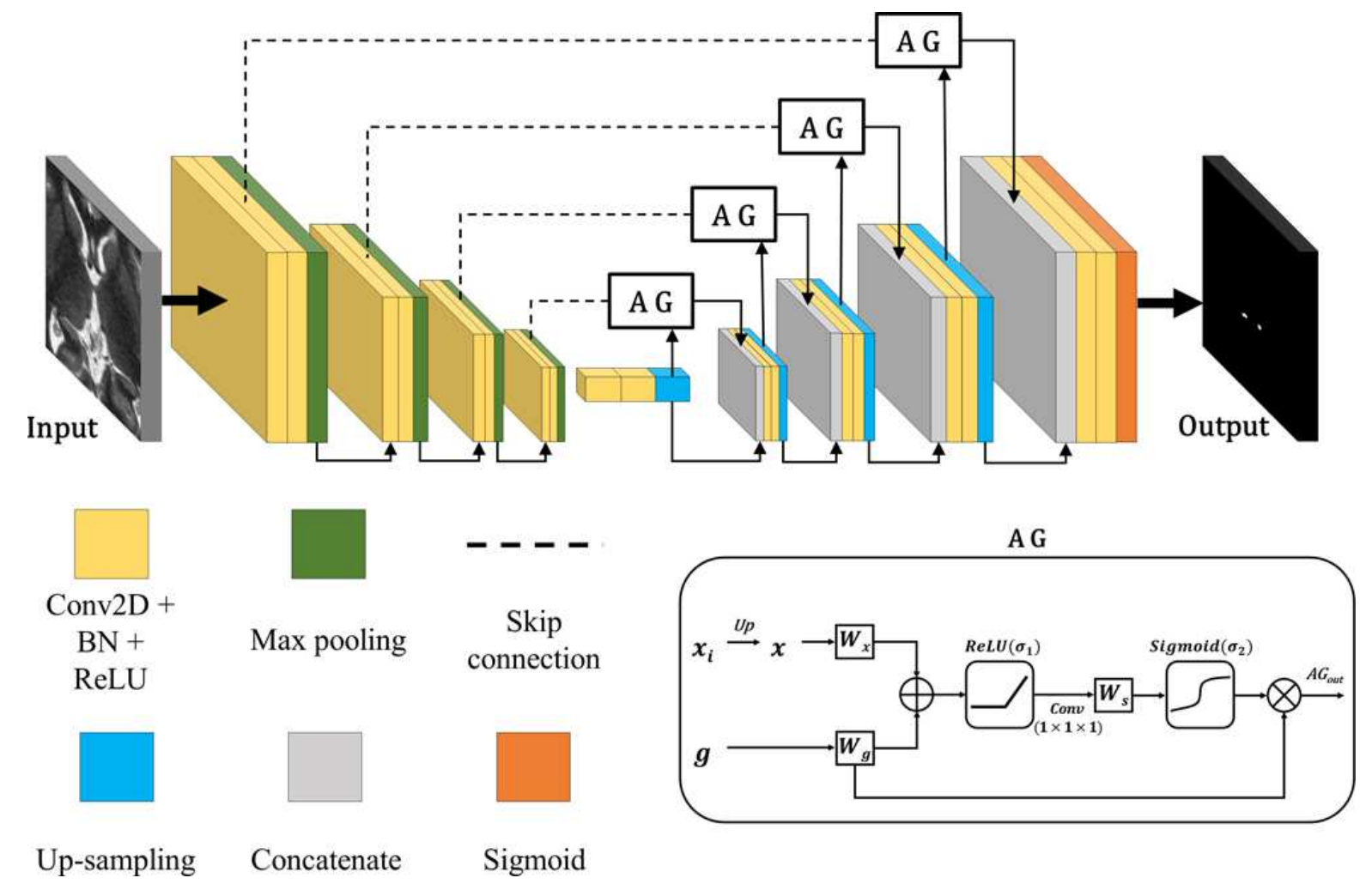

Figure 3. The architecture of the attention gate-based u-net for $\mathrm{Hb}$ segmentation.

Each colored block including the AG process is indicated below the illustration of the network architecture. The significant feature maps were aggregated by the listed convolution operations, including $\mathrm{AG}$, to segment the $\mathrm{Hb}$ regions.

Abbreviations: Conv: convolution; BN: batch normalization; AG: attention gate; ReLU: rectified linear unit; FCN: fully convolutional network; $x$ : the feature maps of the previous layer; $g$ : the skip connected feature maps; $W$ : aggregation procedure of more than one feature with convolutional filters; $W_{s}$ : aggregation procedure of only one feature with a single convolutional filter 


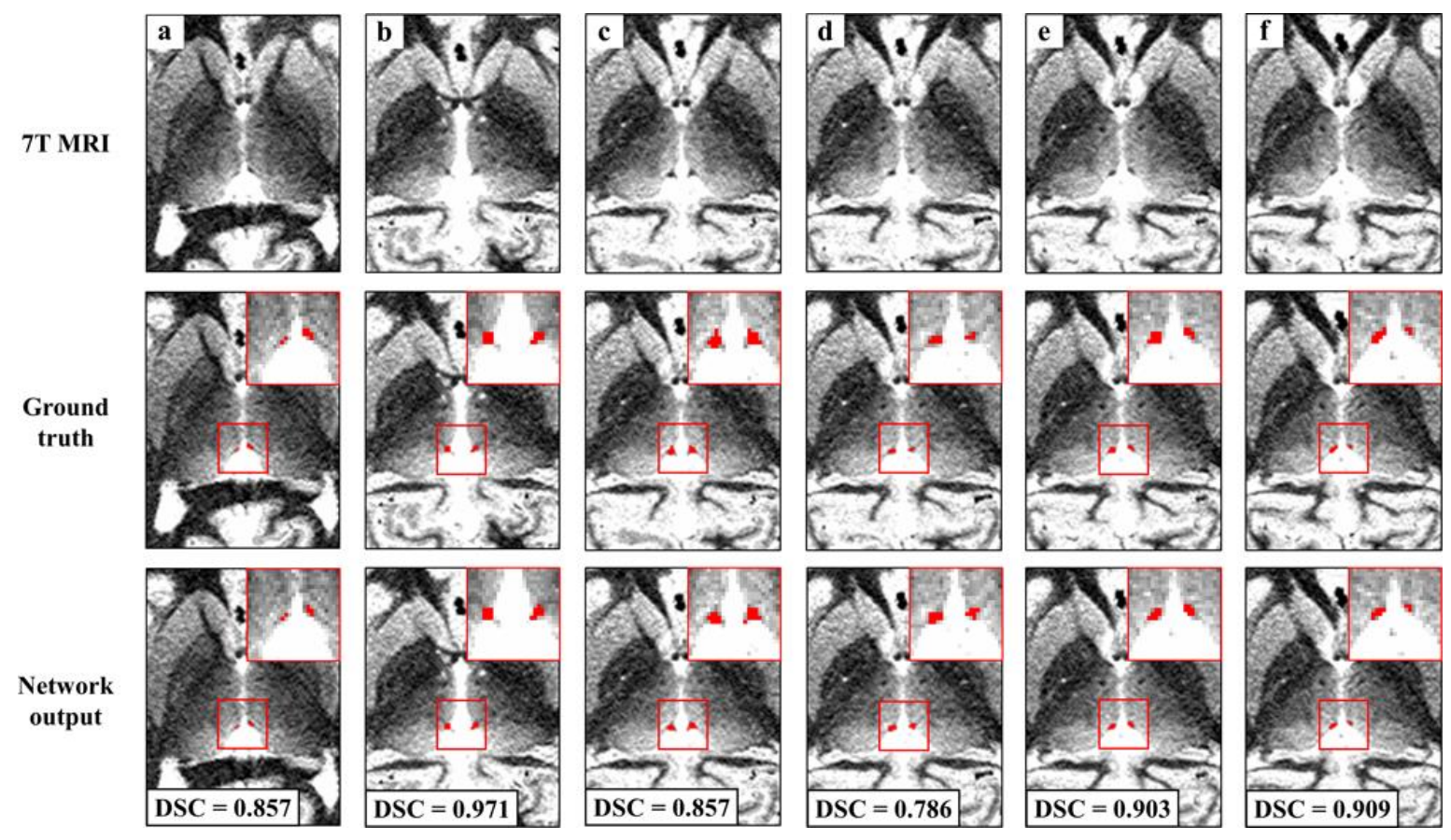

Figure 4. Segmentation results of several test cases $(\mathbf{a}-\mathbf{f})$.

Presented MRIs were set to window level, 1300 and window width, 750 (top row). The ground truth is the intersection of two examiners (middle row). The prediction result is the intersection of the two trained segmentation networks (bottom row). Both ground truth and the automated segmentation results are presented as overlays on cross-sectional 7T MRIs.

Abbreviations: DSC, dice similarity coefficient; MRI, magnetic resonance imaging; 7T, 7 Tesla 


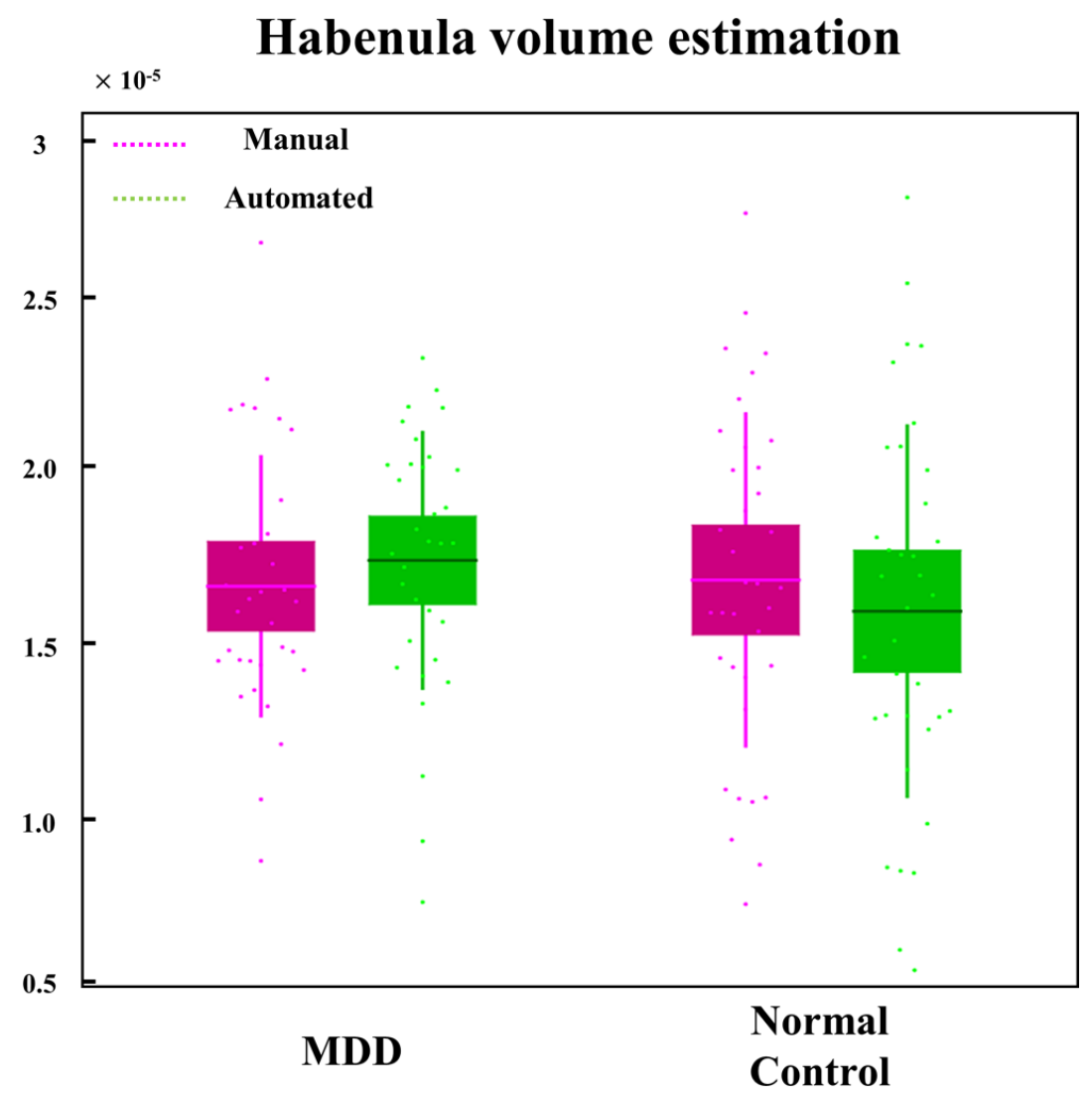

Figure 5. Boxplot of the habenula total volumes normalized by total intracranial volume.

The mean and standard deviation of the normalized $\mathrm{Hb}$ volumes by automatic segmentation and manual segmentation in the MDD (left) and NC (right) groups. Magenta components: estimated $\mathrm{Hb}$ volumes based on the manual segmentation. Green components: estimated $\mathrm{Hb}$ volumes based on the proposed automatic segmentation method.

Abbreviations: $\mathrm{Hb}$, habenula; MDD, major depressive disorder; $\mathrm{NC}$, normal control 


\section{Tables}

Table 1. The performance evaluation of the automatic segmentation results using the proposed network.

\begin{tabular}{cccc}
\hline & Precision & Sensitivity & DSC \\
\hline Fold 1 & $0.846 \pm 0.124$ & $0.901 \pm 0.105$ & $0.861 \pm 0.068$ \\
Fold 2 & $0.870 \pm 0.131$ & $0.875 \pm 0.114$ & $0.860 \pm 0.088$ \\
Fold 3 & $0.887 \pm 0.115$ & $0.865 \pm 0.118$ & $0.863 \pm 0.061$ \\
Fold 4 & $0.872 \pm 0.122$ & $0.839 \pm 0.125$ & $0.845 \pm 0.088$ \\
Fold 5 & $0.868 \pm 0.125$ & $0.843 \pm 0.183$ & $0.831 \pm 0.139$ \\
Average & $\mathbf{0 . 8 6 9} \pm \mathbf{0 . 1 2 4}$ & $\mathbf{0 . 8 6 5} \pm \mathbf{0 . 1 3 4}$ & $\mathbf{0 . 8 5 2} \pm \mathbf{0 . 0 9 4}$ \\
\hline
\end{tabular}

The evaluation results are presented as mean and standard deviation.

Abbreviations: DSC, dice similarity coefficient 
Table 2. The intraclass correlation analysis between automatic and manually segmented habenula volumes.

\begin{tabular}{|c|c|c|c|c|}
\hline & \multicolumn{2}{|c|}{ Habenula volume $^{\mathrm{a}}$} & \multicolumn{2}{|c|}{ Intraclass correlation } \\
\hline & Auto & Manual & $I C C$ & $p$-value \\
\hline \multicolumn{5}{|c|}{ Total participants $(\mathrm{n}=69)$} \\
\hline Total & $16.66 \times 10^{-4} \pm 4.39 \times 10^{-4}$ & $16.77 \times 10^{-4} \pm 4.05 \times 10^{-4}$ & 0.870 & $<0.001$ \\
\hline Left & $8.50 \times 10^{-4} \pm 2.55 \times 10^{-4}$ & $8.63 \times 10^{-4} \pm 2.42 \times 10^{-4}$ & 0.908 & $<0.001$ \\
\hline Right & $8.16 \times 10^{-4} \pm 2.15 \times 10^{-4}$ & $8.14 \times 10^{-4} \pm 2.30 \times 10^{-4}$ & 0.750 & $<0.001$ \\
\hline \multicolumn{5}{|c|}{$\operatorname{MDD}(n=33)$} \\
\hline Total & $17.38 \times 10^{-4} \pm 3.49 \times 10^{-4}$ & $16.68 \times 10^{-4} \pm 3.54 \times 10^{-4}$ & 0.818 & $<0.001$ \\
\hline Left & $8.93 \times 10^{-4} \pm 2.05 \times 10^{-4}$ & $8.72 \times 10^{-4} \pm 1.99 \times 10^{-4}$ & 0.920 & $<0.001$ \\
\hline Right & $8.45 \times 10^{-4} \pm 1.88 \times 10^{-4}$ & $7.96 \times 10^{-4} \pm 2.31 \times 10^{-4}$ & 0.658 & 0.001 \\
\hline \multicolumn{5}{|l|}{$\mathrm{NC}(\mathrm{n}=36)$} \\
\hline Total & $16.00 \times 10^{-4} \pm 5.04 \times 10^{-4}$ & $16.85 \times 10^{-4} \pm 4.52 \times 10^{-4}$ & 0.897 & $<0.001$ \\
\hline Left & $8.11 \times 10^{-4} \pm 2.91 \times 10^{-4}$ & $8.55 \times 10^{-4} \pm 2.79 \times 10^{-4}$ & 0.903 & $<0.001$ \\
\hline Right & $7.89 \times 10^{-4} \pm 2.37 \times 10^{-4}$ & $8.30 \times 10^{-4} \pm 2.31 \times 10^{-4}$ & 0.819 & $<0.001$ \\
\hline
\end{tabular}

${ }^{a}$ Habenula volumes were normalized using total intracranial volume (ICV). Habenula volumes were divided by the ICV for each participant as a normalization process (regional brain volume/ ICV $\times 100 \%$ ) for the subsequent analyses. Normalized habenula volumes are described as mean $\pm \mathrm{SD}$.

Abbreviations: SD, standard deviation; ICC, intraclass correlation coefficient

Significant results are indicated in bold. 


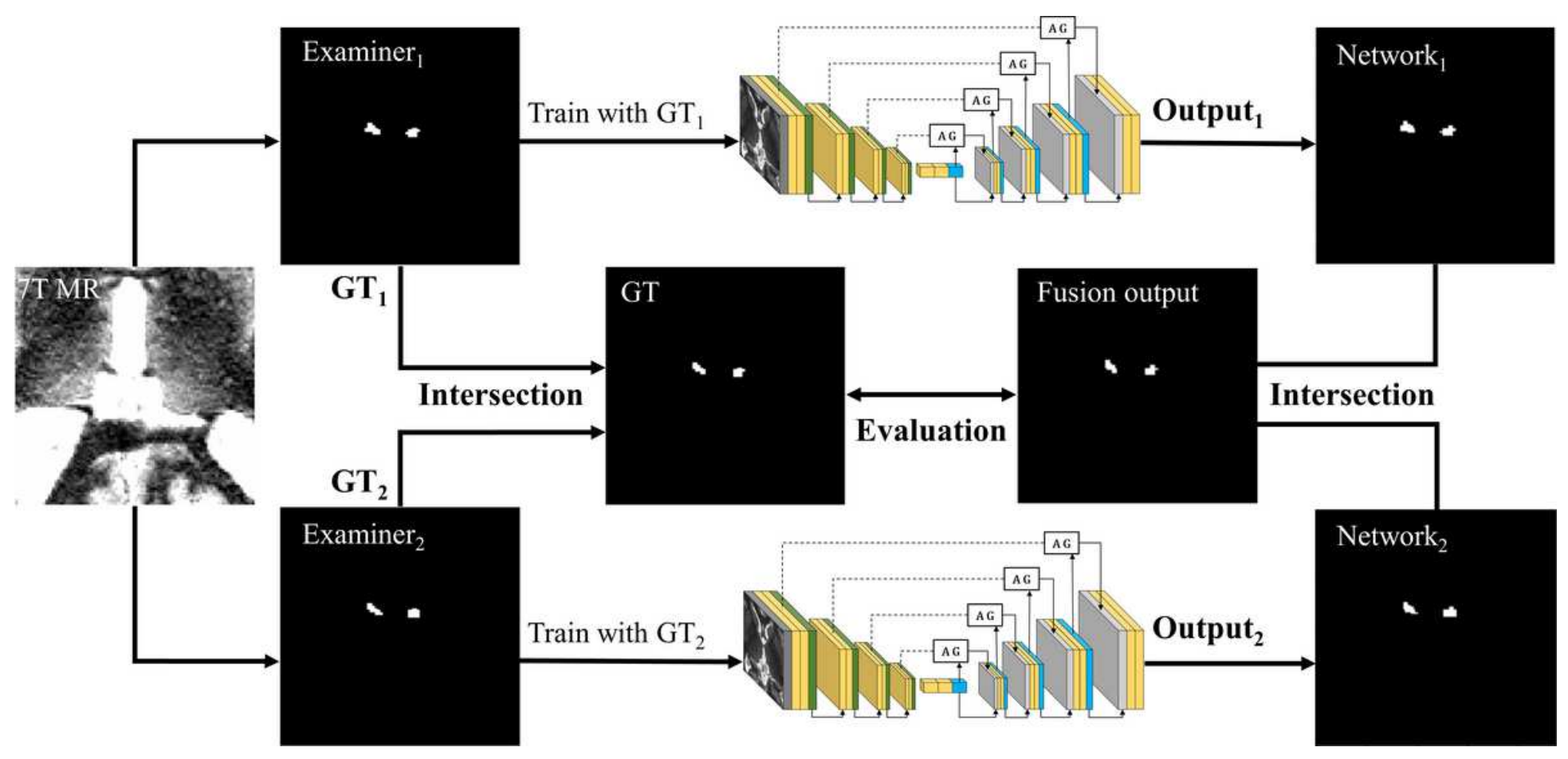

Figure 1

An illustrated overview of the automatic habenula segmentation. In the network training procedure, two manual segmentation masks were used for the training of two networks, and two segmentation results were obtained. The network evaluation was performed by comparing the intersected GT and fusion output. Abbreviations: GT, ground truth; MR, magnetic resonance; 7T, 7 Tesla; AG, attention gate.
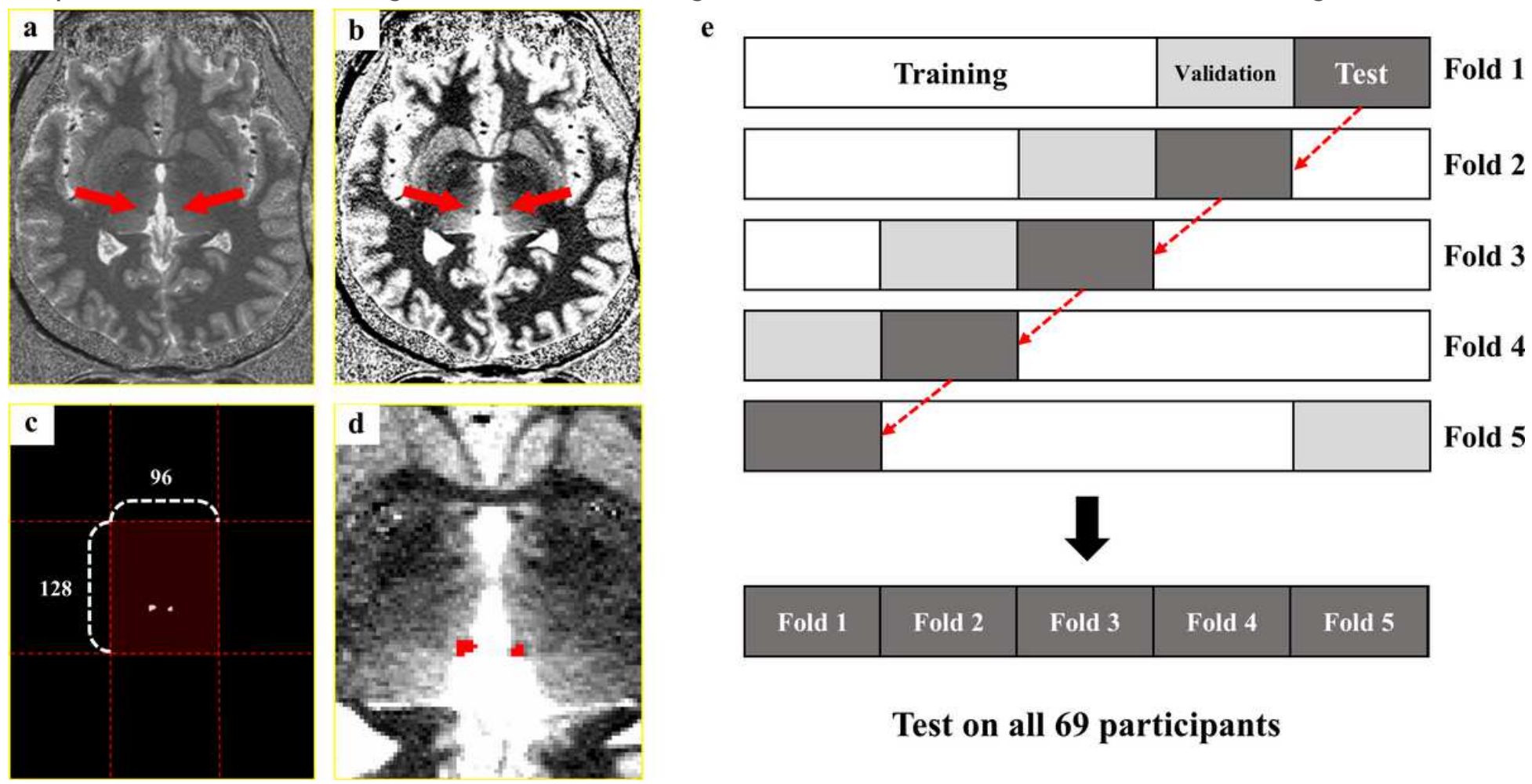

\section{Test on all 69 participants}


Figure 2

Preprocessing procedure of 7T MR images $(a-d)$, and experimental setup (e). (a) Original axial view 7T MRI. (b) Window level (WL) and window width (WW) were set (WL/WW: 1300/750) to observe the Hb. (c) Region of the habenula. (d) Cropped to the specific size. (e) The dataset was divided into 5 folds for testing the segmentation performance on all participants. Abbreviations: MR, magnetic resonance; 7T, 7 Tesla

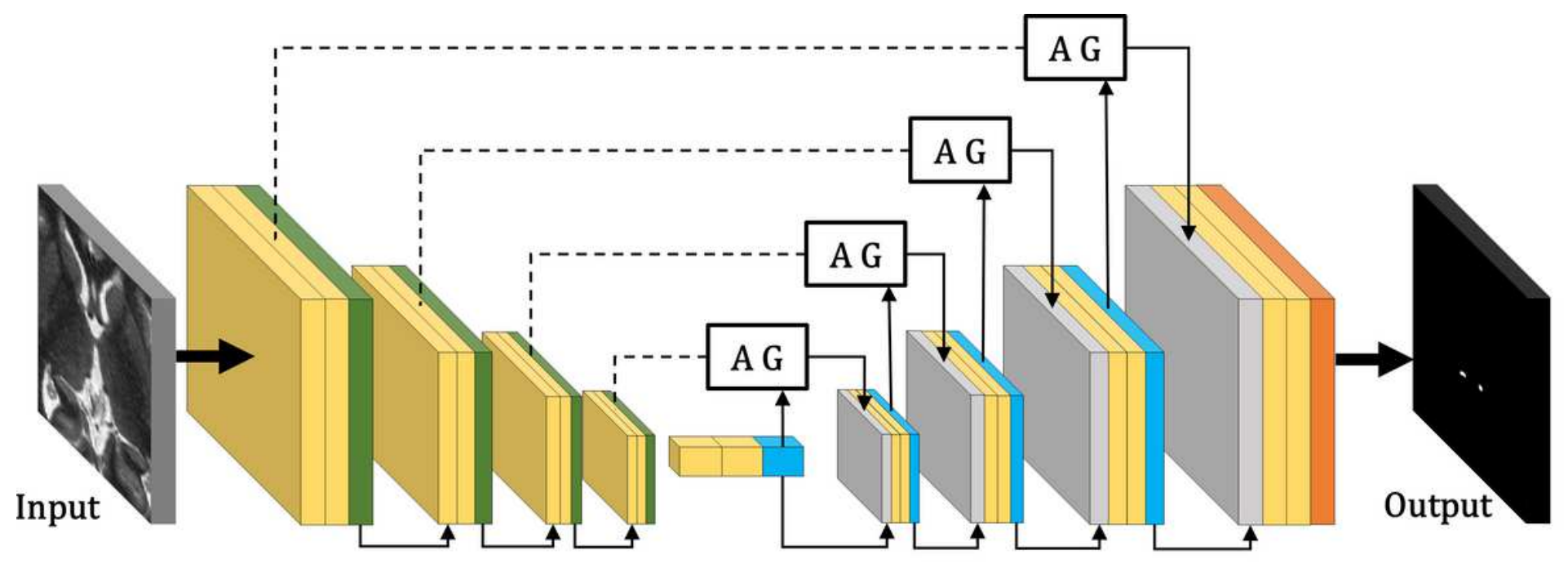

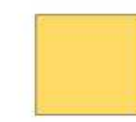

Conv2D + $\mathrm{BN}+$ ReLU

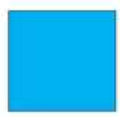

Up-sampling

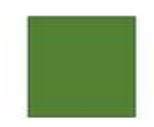

Max pooling

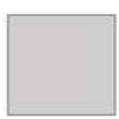

Concatenate

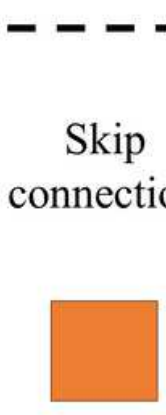

Sigmoid

\section{Figure 3}

The architecture of the attention gate-based u-net for $\mathrm{Hb}$ segmentation. Each colored block including the AG process is indicated below the illustration of the network architecture. The significant feature maps were aggregated by the listed convolution operations, including $\mathrm{AG}$, to segment the $\mathrm{Hb}$ regions. Abbreviations: Conv: convolution; BN: batch normalization; AG: attention gate; ReLU: rectified linear unit; FCN: fully convolutional network; $x$ : the feature maps of the previous layer; $g$ : the skip connected feature maps; W: aggregation procedure of more than one feature with convolutional filters; W_s: aggregation procedure of only one feature with a single convolutional filter 

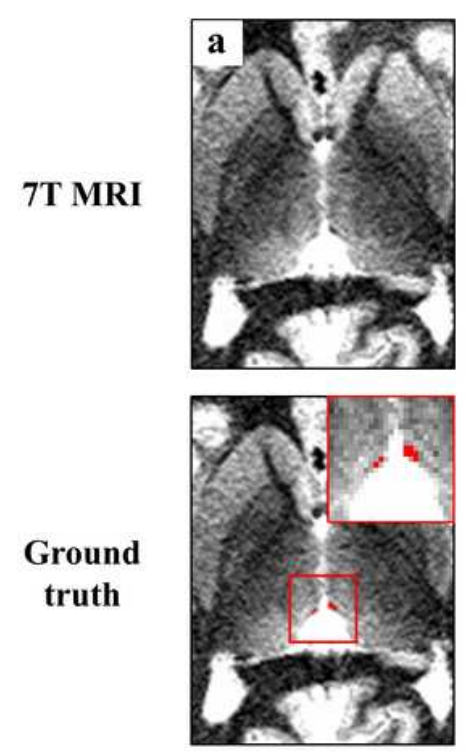

Ground truth

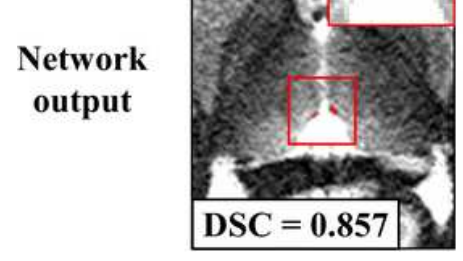

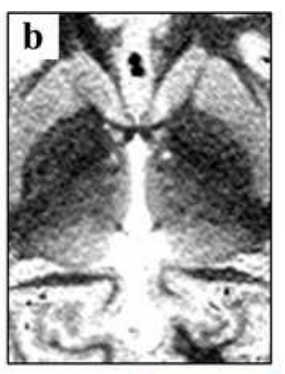
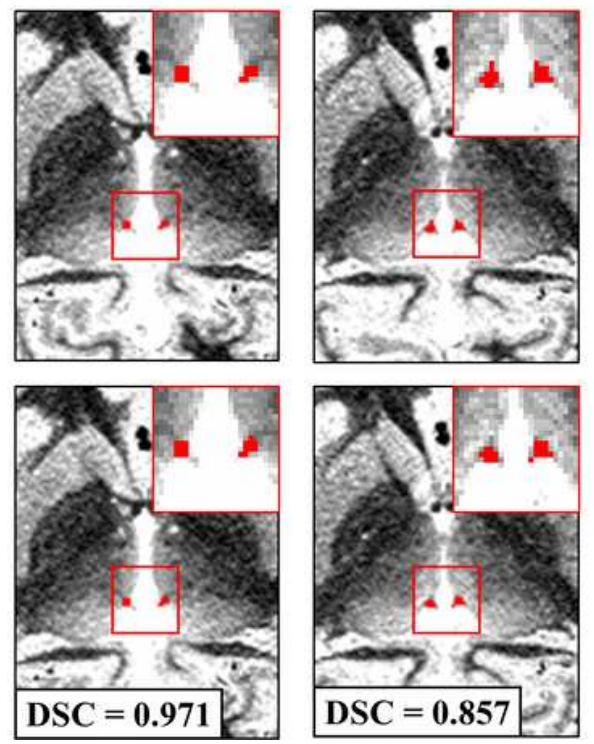
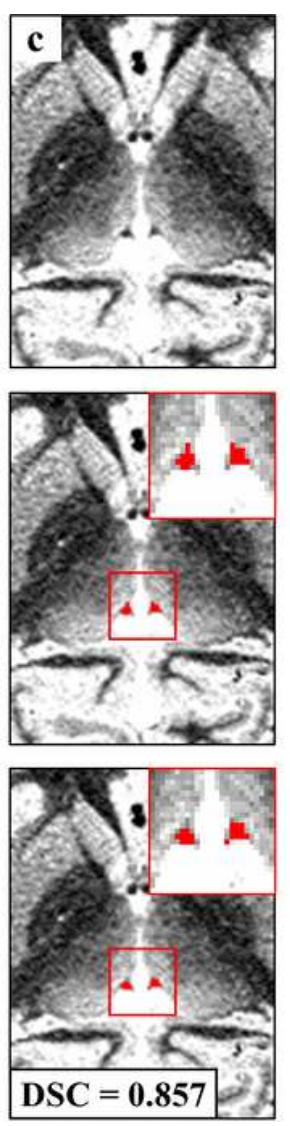
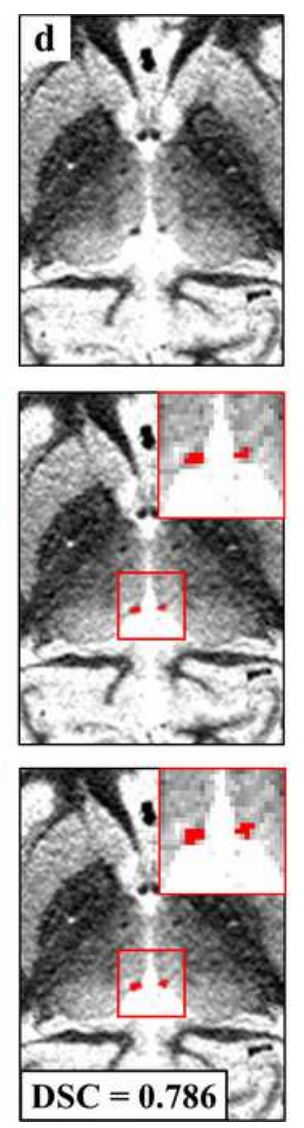
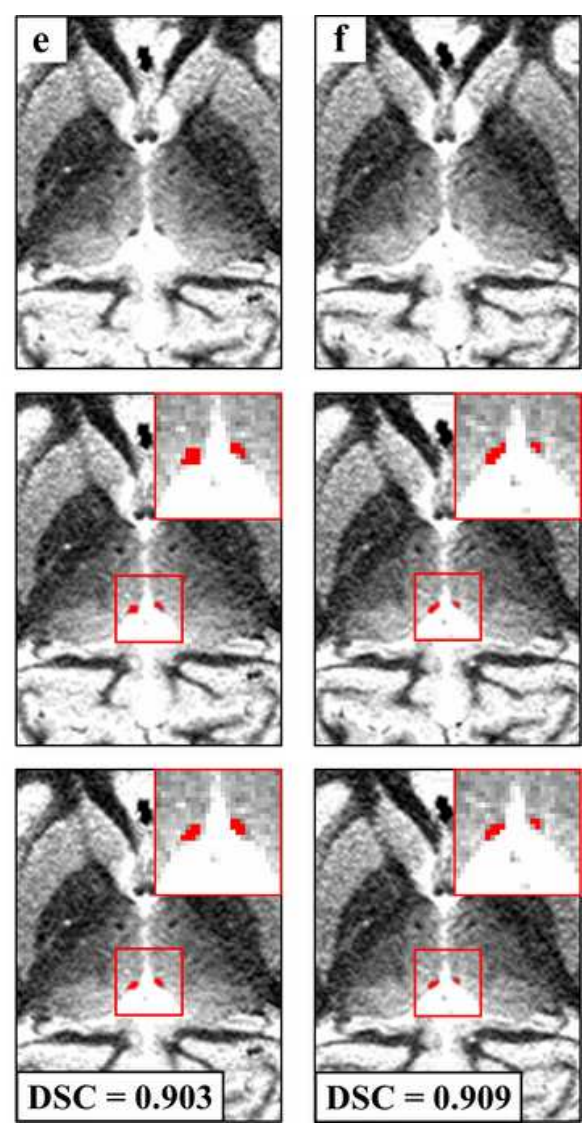

Figure 4

Segmentation results of several test cases $(a-f)$. Presented MRls were set to window level, 1300 and window width, 750 (top row). The ground truth is the intersection of two examiners (middle row). The prediction result is the intersection of the two trained segmentation networks (bottom row). Both ground truth and the automated segmentation results are presented as overlays on cross-sectional 7T MRIs. Abbreviations: DSC, dice similarity coefficient; MRI, magnetic resonance imaging; 7T, 7 Tesla 


\section{Habenula volume estimation}

$\times 10^{-5}$

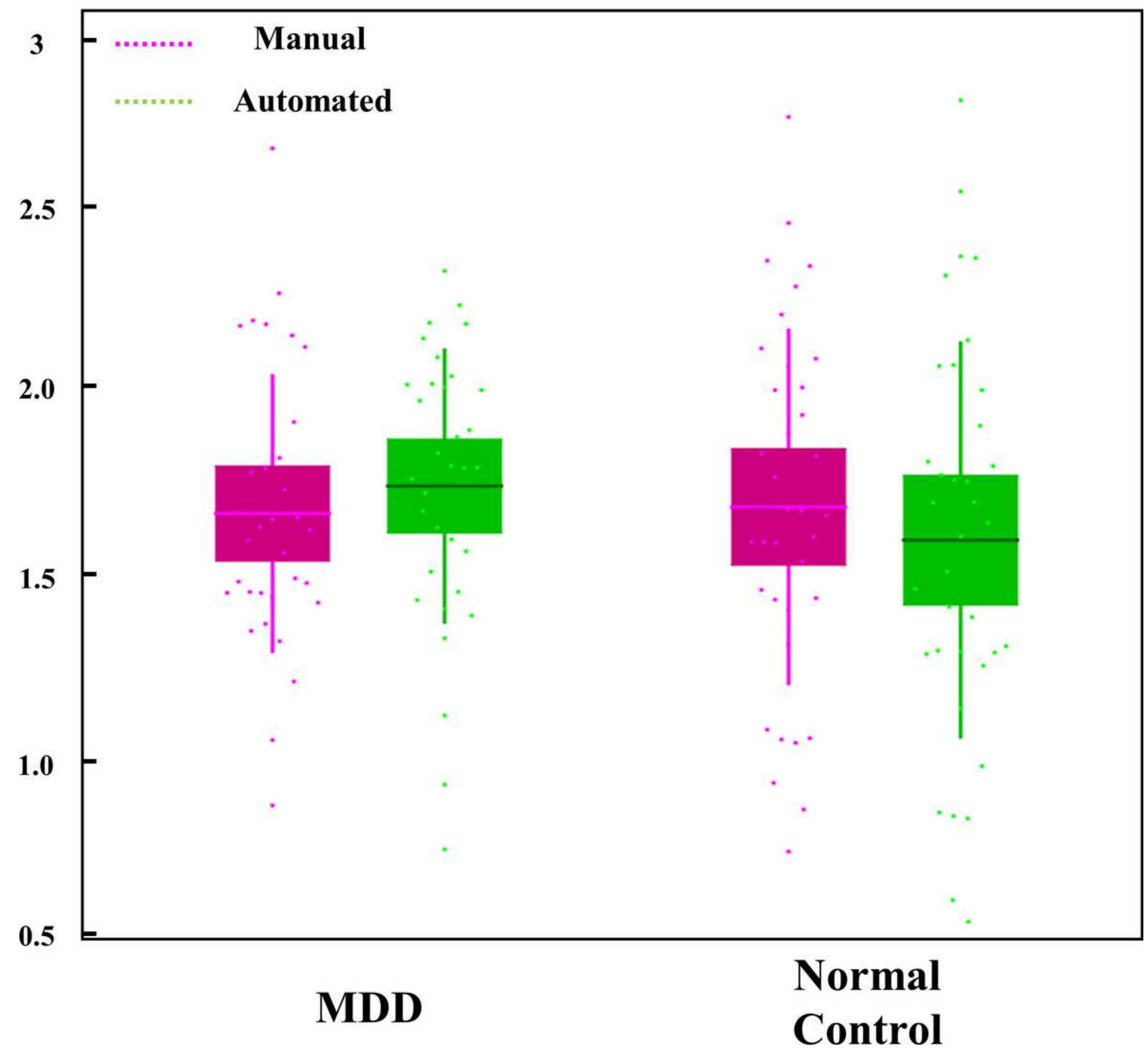

Figure 5

Boxplot of the habenula total volumes normalized by total intracranial volume. The mean and standard deviation of the normalized $\mathrm{Hb}$ volumes by automatic segmentation and manual segmentation in the MDD (left) and NC (right) groups. Magenta components: estimated $\mathrm{Hb}$ volumes based on the manual segmentation. Green components: estimated $\mathrm{Hb}$ volumes based on the proposed automatic segmentation method. Abbreviations: $\mathrm{Hb}$, habenula; MDD, major depressive disorder; NC, normal control 


\section{Supplementary Files}

This is a list of supplementary files associated with this preprint. Click to download.

- Habenulasupplementarymaterials.pdf 